\title{
The next generation of low-cost personal air quality sensors for quantitative exposure monitoring
}

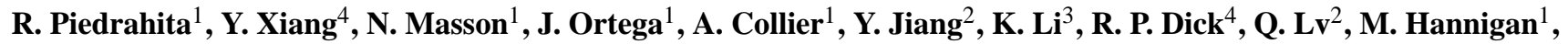 \\ and L. Shang ${ }^{3}$ \\ ${ }^{1}$ University of Colorado Boulder, Department of Mechanical Engineering, 427 UCB, 1111 Engineering Drive, Boulder, \\ CO 80309, USA \\ ${ }^{2}$ University of Colorado Boulder, Department of Computer Science, 1045 Regent Drive, Boulder, CO 80309, USA \\ ${ }^{3}$ University of Colorado Boulder, Department of Electrical Engineering, 425 UCB, 1111 Engineering Drive, Boulder, \\ CO 80309, USA \\ ${ }^{4}$ University of Michigan, Department of Electrical Engineering and Computer Science, 2417-E EECS, 1301 Beal Avenue, \\ Ann Arbor, MI 48109, USA
}

Correspondence to: R. Piedrahita (ricardo.piedrahita@ colorado.edu)

Received: 13 November 2013 - Published in Atmos. Meas. Tech. Discuss.: 12 March 2014

Revised: 29 August 2014 - Accepted: 4 September 2014 - Published: 7 October 2014

\begin{abstract}
Advances in embedded systems and low-cost gas sensors are enabling a new wave of low-cost air quality monitoring tools. Our team has been engaged in the development of low-cost, wearable, air quality monitors (M-Pods) using the Arduino platform. These M-Pods house two types of sensors - commercially available metal oxide semiconductor (MOx) sensors used to measure $\mathrm{CO}, \mathrm{O}_{3}, \mathrm{NO}_{2}$, and total VOCs, and NDIR sensors used to measure $\mathrm{CO}_{2}$. The MOx sensors are low in cost and show high sensitivity near ambient levels; however they display non-linear output signals and have cross-sensitivity effects. Thus, a quantification system was developed to convert the MOx sensor signals into concentrations.

We conducted two types of validation studies - first, deployments at a regulatory monitoring station in Denver, Colorado, and second, a user study. In the two deployments (at the regulatory monitoring station), M-Pod concentrations were determined using collocation calibrations and laboratory calibration techniques. M-Pods were placed near regulatory monitors to derive calibration function coefficients using the regulatory monitors as the standard. The form of the calibration function was derived based on laboratory experiments. We discuss various techniques used to estimate measurement uncertainties.
\end{abstract}

The deployments revealed that collocation calibrations provide more accurate concentration estimates than laboratory calibrations. During collocation calibrations, median standard errors ranged between 4.0-6.1 ppb for $\mathrm{O}_{3}, 6.4$ $8.4 \mathrm{ppb}$ for $\mathrm{NO}_{2}, 0.28-0.44 \mathrm{ppm}$ for $\mathrm{CO}$, and $16.8 \mathrm{ppm}$ for $\mathrm{CO}_{2}$. Median signal to noise ( $\mathrm{S} / \mathrm{N}$ ) ratios for the M-Pod sensors were higher than the regulatory instruments: for $\mathrm{NO}_{2}$, 3.6 compared to 23.4 ; for $\mathrm{O}_{3}, 1.4$ compared to 1.6 ; for $\mathrm{CO}$, 1.1 compared to 10.0; and for $\mathrm{CO}_{2}, 42.2$ compared to 300 500. By contrast, lab calibrations added bias and made it difficult to cover the necessary range of environmental conditions to obtain a good calibration.

A separate user study was also conducted to assess uncertainty estimates and sensor variability. In this study, $9 \mathrm{M}$ Pods were calibrated via collocation multiple times over 4 weeks, and sensor drift was analyzed, with the result being a calibration function that included baseline drift. Three pairs of M-Pods were deployed, while users individually carried the other three.

The user study suggested that inter-M-Pod variability between paired units was on the same order as calibration uncertainty; however, it is difficult to make conclusions about the actual personal exposure levels due to the level of user engagement. The user study provided real-world sensor drift data, showing limited CO drift (under $-0.05 \mathrm{ppm}^{-1 a y}{ }^{-1}$ ), and higher for $\mathrm{O}_{3}\left(-2.6\right.$ to $\left.2.0 \mathrm{ppb} d a y^{-1}\right), \mathrm{NO}_{2}(-1.56$ to 
$\left.0.51 \mathrm{ppb} \mathrm{day}^{-1}\right)$, and $\mathrm{CO}_{2}\left(-4.2\right.$ to $\left.3.1 \mathrm{ppm} \mathrm{day}^{-1}\right)$. Overall, the user study confirmed the utility of the M-Pod as a lowcost tool to assess personal exposure.

\section{Introduction}

\subsection{Background and motivation}

Health effects such as asthma, cardio-pulmonary morbidity, cancer, and all-cause mortality are directly related to personal exposure of air pollutants (EPA ISA Health Criteria, 2010, 2013a, b). To comply with the U.S. Clean Air Act, state monitoring agencies take ongoing measurements in centralized locations that are intended to represent the conditions normally experienced by the majority of the population. Because these measurements require sophisticated, costly, and powerintensive equipment, they can only be taken at a limited number of sites. Depending on the pollutant, individual, and location, this can lead to misleading personal exposure assessments (HEI, 2010). Low-cost, portable, and autonomous sensors have the potential to take equivalent measurements while more effectively capturing spatial variability and personal exposure. Thus, we set out to survey such sensors, analyze their performance, and understand the feasibility of using them. We describe the M-Pod hardware and quantification system, and personal exposure results in greater detail below.

\subsection{Low-cost portable air pollution measurement techniques}

Quantitative measurements of pollutant concentrations generally require techniques to be sensitive at ambient concentrations and unique to that particular compound (in other words, free from interference from other pollutants). Numerous techniques currently exist (including several EPA approved methods); rather than provide an exhaustive report of all available measurement techniques, we provide brief descriptions of the various techniques, along with their measurements, costs, and potential.

\subsubsection{Carbon monoxide}

Federal Reference Method (FRM) measurements of CO are made using infrared absorption instruments, which use $\sim 200 \mathrm{~W}$ power, cost $\sim$ USD $15000-20000$, and require frequent calibrations and quality control checks (EPA Quality Assurance Handbook Vol. II, 2013). By comparison, metal oxide semiconductor (MOx) sensors often cost USD 5-15 and require less than $1 \mathrm{~W}$ of power. One example of this kind of device is the SGX 5525 sensor used for CO measurements that uses approximately $\sim 80 \mathrm{~mW}$ power. MOx sensors have fast responses, low detection limits, and require simple measurement circuitry. However, they can have high cross-sensitivities to other reducing gases, and can be poisoned by certain gases or high doses of target gases.

The typical reducing gas MOx sensor uses a heated tinoxide $n$ type semi-conductor surface, on which oxygen can react with reducing gases, thus freeing electrons in the semiconductor. This lowers the electrical resistance proportional to the concentration of the reducing gas (Moseley, 1997). These sensors suffer from cross-sensitivities to temperature, humidity, and other pollutants. Korotcenkov (2007) provides a comprehensive review of MOx materials and their characteristics for gas sensing, while Fine et al. (2010) and Bourgeois et al. (2003) review the use of MOx sensors and arrays in environmental monitoring.

As compared to traditional monitors, electrochemical sensors are relatively low in cost, $\sim$ USD 50-100, and have been used in multiple studies that required low power sensors for measuring CO (Milton and Steed, 2006; Mead et al., 2013). These sensors exhibit high sensitivity, low detection limit (sub-ppm for some models), fast response, low cross-sensitivity, and consume power in the hundreds of $\mu \mathrm{W}$ range. However, they have more complicated and expensive measurement circuitry, are susceptible to poisoning, have a shorter life span (generally 1-3 years), more expensive than MOx, and are generally larger in size than MOx.

\subsubsection{Ozone}

FRM measurements of $\mathrm{O}_{3}$ are made using the principle of chemiluminescence (EPA ISA Health Criteria, 2013a). Chemiluminescence instruments typically cost USD 1000020000 and use approximately $1 \mathrm{~kW}$. A Federal Equivalence Method uses UV absorption to measure an $\mathrm{O}_{3}$ concentration. Such instruments have prices in the low USD 1000s.

MOx $\mathrm{O}_{3}$ sensors have been commercialized and can cost anywhere in the range of $\sim$ USD 5-100, with power consumption as low as $90 \mathrm{~mW}$. Aeroqual has commercialized a tungsten oxide semiconductor sensor board. Power consumption is $2-6 \mathrm{~W}$, and this material is reported to have less cross-sensitivity and calibration drift than other MOx materials (Williams et al., 2009, 2013). As discussed in more detail later, we refer to drift when discussing changes to the calibration function coefficients over time, given recalibrations under the same conditions. Electrochemical sensors are also available with reported noise of $4 \mathrm{ppb}$, but with significant cross-sensitivity to $\mathrm{NO}_{2}$ (Alphasense, 2013a).

\subsubsection{Nitrogen oxides $\left(\mathrm{NO}_{\mathrm{x}}\right)$}

FRM measurements of $\mathrm{NO}_{\mathrm{x}}$ are made using the chemiluminescence reaction of $\mathrm{O}_{3}$ with $\mathrm{NO}$ along with the catalytic reduction of $\mathrm{NO}_{2}$ to NO (EPA ISA Health Criteria, 2013b). These instruments typically cost USD 10000-20000 and consume approximately $1 \mathrm{~kW}$ power. $\mathrm{NO}_{2}$ can also be measured with electrochemical sensors ( $\sim$ USD $80-210$ ) (Alphasense, 2013b; SGX 
Sensortech (http://www.sgxsensortech.com/) and MOx sensors ( USD 4-54) SGX Sensortech; Synkera (www. Synkerainc.com); Figaro (http://www.figarosensor.com/)).

\subsubsection{Carbon dioxide $\left(\mathrm{CO}_{2}\right)$}

$\mathrm{CO}_{2}$ is the primary anthropogenic greenhouse gas, as well as a proxy for assessing ventilation conditions in indoor environments. Elevated concentrations have been found to affect decision-making and exam performance (Satish et al., 2012). Portable non-dispersive infrared (NDIR) carbon dioxide sensors are precise, easy to calibrate, easy to integrate into a mobile sensing system (Yasuda et al., 2012), and are commercially available for under USD 100 to a few hundred USD. The sensors operate by emitting a pulse of infrared radiation across a chamber. A detector at the other end of the chamber measures light intensity. Absorption of light by $\mathrm{CO}_{2}$ accounts for the difference between expected and measured intensity. Interference can occur due to absorption by water vapor and other gasses and drift can occur due to changes in the light source (Zakaria, 2010). Electrochemical sensors are also available to measure $\mathrm{CO}_{2}$. They are inexpensive and have low power requirements, but generally have slower response times, shorter life spans, and are more susceptible to poisoning and drift than NDIR-type sensors.

\subsection{Instruments for personal air quality monitoring}

Personal exposure has been characterized extensively using filter samplers, particle counters, and sorbent tubes. These methods can provide simple, accurate, and comprehensive speciation results; however, because each filter or adsorbent tube typically samples for durations of a day or more, important time series information is often lost when using these methods. Relatively recent sampling techniques allow for higher time-resolution personal measurement of pollutants.

Electrochemical sensors have been used to monitor $\mathrm{CO}$ in many works, including Kaur et al. (2007), Mead et al. (2013), Honicky et al. (2008), and Milton and Steed (2006). Shum et al. (2011) developed a wearable $\mathrm{CO}, \mathrm{CO}_{2}$, and $\mathrm{O}_{2}$ monitor. Mead et al. (2013) and Honicky et al. (2008), using electrochemical and MOx sensors, also monitored both $\mathrm{O}_{3}$ and $\mathrm{NO}_{\mathrm{x}}$ in the works listed above. Williams et al. (2009) developed and deployed a portable tungsten oxide-based $\mathrm{O}_{3}$ sensor and $\mathrm{NO}_{2}$ sensor. Hasenfratz et al. (2012) also monitored $\mathrm{O}_{3}$ in a train-mounted instrument study using metal oxide semiconductor sensors. Hasenfratz's work tested collaborative calibration performance, in which sensor nodes were periodically co-located to check and improve calibrations. De Vito et al. (2009) developed a wearable system to measure CO, $\mathrm{NO}_{2}$, and $\mathrm{NO}_{\mathrm{x}}$, using MOx sensors, and employed machine learning techniques for calibration and quantification.

Tsow et al. (2009) developed wearable monitors to measure benzene, toluene, ethyl-benzene and xylene at ppb levels. The measurement is based on a MEMS tuning fork design that provides good selectivity and low detection limits, but the device is not yet commercially available. Electronic nose systems for sensing VOCs are commercially available, often designed to detect specific gas mixtures from processes. Such systems use a variety of sensing techniques, including those mentioned above, as well as polymer-coated sensors, mass spectrometry, ion mobility spectrometry, and gas chromatography, among others (Gardner and Bartlett, 1994; Röck et al., 2008). Much potential remains to be exploited in this area, as there has been difficulty in transferring laboratory success to the field (Marco, 2014).

These models and most real-time personal exposure monitors are currently too expensive to be truly ubiquitous. Fortunately, advancements in technology and increasing concern about air quality in many regions have produced a wave of low-cost personal exposure instruments. Reliable results are needed for users of these low-cost monitors before they take action to reduce their exposure. We describe our novel quantification system that includes collocation calibration (sometimes referred to as normalization), modeling of sensor responses with environmental variables, and uncertainty estimation for these measurements. We demonstrate this quantification system by presenting results from a user study where six users wore monitors for 10-20 days.

\section{Methods}

\subsection{MAQS - Mobile Air Quality Sensing System}

The key requirements for our mobile sensing system included wearability and portability, low-cost, multi-pollutant, wireless communication, and enough battery life to wear for an entire day. The goal was for our system to sense as many National Ambient Air Quality Standards (NAAQS) criteria pollutants at typical ambient concentrations as possible. The result of our development effort is the M-Pod, shown in Fig. 1.

The M-Pod collects, analyzes, and shares air quality data using the Mobile Air Quality Sensing (MAQS) system (Jiang et al., 2011). An Android mobile phone application, MAQS3 (Mobile Air Quality Sensing v.3), pairs with the M-Pod via Bluetooth, and the M-Pod data is transmitted to the phone periodically. The data is then sent to a server for analysis. A web-based analysis and GIS visualization platform can access new data from the server. Wi-Fi fingerprints can also be used to identify an M-Pod's indoor locations (Jiang et al., 2012). The M-Pod has also been configured to operate with another environmental data collection app, AirCasting (http://aircasting.org/).

Each M-Pod houses four MOx sensors to measure CO, total VOCs, $\mathrm{NO}_{2}$, and $\mathrm{O}_{3}$ (SGX Corporation models MiCS5525, MiCS-5121WP, MiCS-2710, and MiCS-2611), an NDIR sensor (ELT, S100) to measure $\mathrm{CO}_{2}$, a fan to provide steady flow through the device (Copal F16EA-03LLC), 

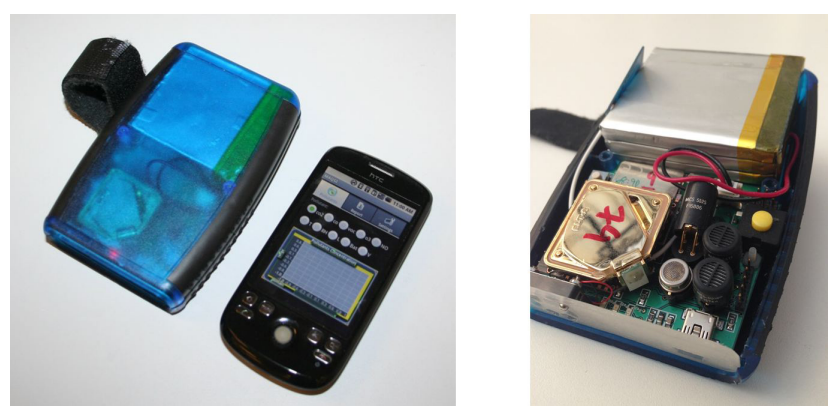

Figure 1. The M-Pod and the accompanying MAQS3 phone application.

a light sensor, and a relative humidity and temperature sensor (Sensirion, SHT21). Socket-mount MOx sensors were preferred over surface-mount sensors because of the difficulty replacing the surface-mount sensors and possible poisoning of the surface-mount sensors due to soldering (hot-air reflow).

\subsection{Calibration system}

MOx sensors represent the lowest cost sensing solution but hold significant quantification challenges. MOx sensor responses are non-linear with respect to gas concentration, and are affected by ambient temperature and humidity (Sohn et al., 2008; Barsan and Weimar, 2001; Delpha et al., 1999; Romain et al., 1997; Marco, 2014).

Baseline drift and changes in sensitivity over time are also common. As will be discussed further later, we define drift as changes in sensor baseline over time. More specifically, we identify two factors contributing to temporal drift: predictable drift due to changes in the heater output, and unpredictable drift due to poisoning or irreversible bonding to the sensor surface (Romain and Nicolas, 2010). As such, using MOx sensors quantitatively requires that a model be developed which not only characterizes the relationship between sensor resistance and gas concentration, but also includes the impacts of these other variables and sensor characteristics. Below we describe our calibration system and strategies for overcoming these challenges.

Our calibration system uses automated mass flow controllers (MFCs, Coastal Instruments FC-2902V) and solenoidal valves to inject specific mixtures of gas standards into a Teflon-coated aluminum chamber that is equipped with temperature and relative humidity control. The $\mathrm{CO}$ and $\mathrm{NO}_{2}$ used were premixed certified gas standards, while the $\mathrm{CO}_{2}$ and air were industrial and zero-grade, respectively. Constant gas flows were administered using the mass flow controllers, which were calibrated prior to the M-Pod calibrations. Custom LabVIEW software (LabVIEW 2011) and Labjack data acquisition devices (LabJack U3-LV) were used for instrument control and data logging.
The $\mathrm{CO}$ and $\mathrm{NO}_{2}$ sensors were calibrated for changes in both temperature and humidity. By contrast, the $\mathrm{CO}_{2}$ sensors were only calibrated for temperature, as they show a small non-linear response to temperature. While humidity effects have been reported for NDIR sensors in other studies (Yasuda et al., 2012), previous calibrations in our lab showed that it is not a significant issue in this case. Temperature is controlled using a heat lamp and by performing calibrations inside a refrigerated chamber. Routing a portion of the airflow through deionized water controls relative humidity, using a 3-way valve.

The M-Pods were placed in a carousel type enclosure that holds 12 M-Pods and allows for uniform gas diffusion into each pod (Supplement Fig. S2). The carousel, which is made of steel with a polycarbonate lid, has a volume of $2.2 \mathrm{~L}$, and conditions reach $T_{90}$ steady state in $120 \mathrm{~s}$ or less using our selected flow rate of $4.3 \mathrm{Lpm}$. For calibrations, the carousel is either placed inside of the Teflon coated chamber, or in a refrigerator, depending on the desired temperature level.

Calibrations were performed after sensors operated continuously for at least a week. Performance of these sensors for 1 week was important to ensure adequate sensor warmup and stabilization time. Specifically, warm-up time allows for stabilization of the semiconductor heating element, which can drift substantially in the first week (Masson et al., 2014). After this period, warm-up times when the sensor is simply heating up to operating temperature are shorter, on the order of $10 \mathrm{~min}$. Because each calibration run consisted of different gas concentrations, temperature and humidity set points, sensors were held at each state for periods of $15 \mathrm{~min}$ to allow them to reach steady state. The last $30 \mathrm{~s}$ of each $15 \mathrm{~min}$ period were averaged, and these points were used for the calibration. Administered concentrations depend on the expected deployment environment, and in this case stepped from 0-1.0-2.0$4.2 \mathrm{ppm}$ for $\mathrm{CO}$, and 0-500-1075-1650 ppm for $\mathrm{CO}_{2}$. Combinations of environmental conditions for these calibrations included M-Pod temperatures of approximately 302 and $317 \mathrm{~K}$, and for $\mathrm{CO}$ calibration additional relative humidity levels of 20 and $60 \%$ were employed. A CO calibration time series and surface used after one deployment at CAMP is shown in Figs. S3 and S4 in the Supplement.

Initially, the sensors were calibrated by mounting them on large arrays, but we found that the sensor response is highly dependent on the position in the array and air-flow conditions. The convective cooling of the sensors is thus an important variable, as will be discussed further later. To ensure that calibration temperature and flow conditions about each sensor are the same as during operating conditions, they are calibrated in their individual M-Pods.

\subsection{Development of quantification models}

To simplify the inter-comparison of MOx sensors (which are often heterogeneous from sensor to sensor; Romain and Nicolas, 2010), it is common practice to normalize a sensor's 
resistance by a reference resistance, $R_{0}$. The reference resistance is the sensor's unique response to a given environment, for example, cleans air at $25^{\circ} \mathrm{C}$, standard atmospheric pressure, and $20 \%$ relative humidity. As such, a sensor quantification model relates $R_{\mathrm{S}} / R_{\mathrm{O}}$ to concentration, temperature and humidity. Other works have developed procedures for this for different sensors and applications, using a variety of techniques. For example, De Vito et al. (2009) used a multivariate approach with automatic Bayesian regularization to limit the effects of cross-sensitivity. Numerous works have also used machine-learning techniques such as neural networks to determine concentration values and/or identify mixtures (Kamionka et al., 2006; Zampolli et al., 2004; Sundgren et al., 1991; Wolfrum et al., 2006) and identify pollution sources. However, to our knowledge, a parametric regression-based model has yet to be developed for these specific sensors. We believe this type of model is preferable for ease of implementation. Comparing system performance with an aforementioned machine learning-based approach is a logical next step for this research.

Two sensor models were chosen for the majority of the analysis conducted thus far: the MiCS-5121WP CO/VOC sensor and MiCS-5525 CO sensor (both manufactured by SGX Sensortech). The VOC sensor was chosen because of our strong initial interest in indoor air pollution. The MiCS5525 was the logical next step because it has the same semiconductor sensor surface as the MiCS-5121, but with an activated charcoal pre-filter. Both lab data and ambient collocation data were used to convert sensor signal to concentration. In lab experiments, the sensors were calibrated in the Tefloncoated chamber. The chamber and calibration system are described in detail in the Supplement. The model derived from this data was then applied to each M-Pod CO sensor used in the collocation. Our results show that the $\mathrm{CO}, \mathrm{NO}_{2}$ and $\mathrm{O}_{3}$ MOx sensors can detect ambient concentrations in Colorado when frequently calibrated. For context, ambient concentrations of the criteria pollutants in Colorado are usually NAAQS compliant. $\mathrm{O}_{3}$ is the only pollutant with occasional violations at some local monitoring sites (CDPHE Annual Data Report, 2012).

Figure 2 illustrates the MiCS-5525 CO sensor response to changing temperature at various concentrations of $\mathrm{CO}$. Although humidity can have a substantial effect on sensor response, we found that with these sensors the expected range of absolute humidity has a lesser effect on signal response than the effect of the expected temperature range. Therefore, absolute humidity was held constant so as to simplify the procedure and minimize the degrees of freedom within the model response. We later add a humidity term in the collocation calibration analysis to improve model performance. From experimental observation, the sensor response appears to change linearly with respect to temperature for a given $\mathrm{CO}$ concentration between concentrations of 0 and $2.8 \mathrm{ppm}$. The slope and intercept of the linear temperature trends also appear to decrease with increasing $\mathrm{CO}$ concentration.

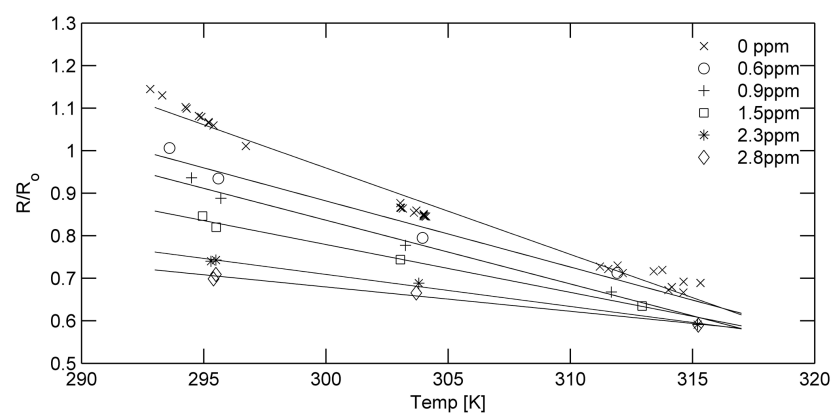

Figure 2. MiCS-5525 CO sensor response to various CO concentrations while held at different ambient temperatures.

Equation (1) was chosen as the best fit for the observed sensor response to $\mathrm{CO}$ concentration and temperature. A third term of the same form was added to the model to account for changes in absolute humidity $(H)$.

$\frac{R_{\mathrm{S}}}{R_{\mathrm{o}}}=f(C)(T-298)+g(C)+h(C) H$

In this model, $f(C)$ describes the change in temperature slope with respect to pollutant concentration; $g(C)$ describes the change in resistance in dry air at $298 \mathrm{~K}$ due to concentration; and $h(C)$ describes the change in absolute humidity slope with respect to concentration. The terms $f(C), g(C)$, and $h(C)$ were chosen to be of the form $p_{1} \exp \left(C p_{2}\right)$.

This model form performed well for all MOx sensors used, but is computationally challenging to work with because it is not algebraically invertible. Instead, we used a second-order Taylor approximation for this model (Kate, 2009). However, an even simpler model in temperature, absolute humidity, and concentration (Eq. 2) was found to perform similarly in many cases. The comparable performance of the models is likely due to the low variation in $\mathrm{CO}$ concentration observed throughout the field experiments. Though we did not perform the same lab calibration tests with the $\mathrm{NO}_{2}$ and $\mathrm{O}_{3}$ sensors, we found that in collocation calibrations, Eqs. (2) and (3) also fit the data comparably to the model in Eq. (1).

$$
\frac{R_{\mathrm{S}}}{R_{\mathrm{o}}}=p_{1}+p_{2} C+p_{3} T+p_{4} H
$$

In cases with longer time series and multiple calibrations, a time term, $p_{5} t$, was added to correct for temporal drift.

$$
\frac{R_{\mathrm{S}}}{R_{\mathrm{O}}}=p_{1}+p_{2} C+p_{3} T+p_{4} H+p_{5} t
$$

Equation (3) was used throughout the results unless otherwise noted.

We determined concentration uncertainty by propagating the error in the calibration model through the inverted calibration function (NIST Engineering Statistics Handbook 2.3.6.7.1). The calculation included co-variance terms, but 
did not include the propagated uncertainty of the temperature, humidity, nor voltage measurements, as those are expected to be insignificant relative to the other sources of error. The calculated uncertainty does not directly account for sources of error such as convection heat loss or crosssensitivities that may be seen in field measurements but not during calibration. Convective heat loss due to changes in airflow through the M-Pod are a concern with any system using passive aspiration, as has been shown by Vergara et al. (2013). Collocation calibration should account for some cross-sensitivity effects since there is simultaneous exposure to a wide array of environmental conditions. Some sources of error are still not accounted for though, such as transient temperature effects due to convection. Such effects are likely more substantial when users carry the M-Pod than during a collocation, due to the user's motion and activity.

To explore the validity of this uncertainty propagation, we employed duplicate M-Pods during a user study. For this user study data, when there were duplicate M-Pod measurements but no reference monitors, we used two additional methods to explore uncertainty, the average relative percent difference (ARPD), and the pooled pairwise standard deviation of the differences $\left(\mathrm{SD}_{\text {diff }}\right)$ (Table 3$)$. These formulas are defined as follows:

$\mathrm{SD}_{\text {diff }}=\sqrt{\frac{1}{2 n} \sum_{i=1}^{n}\left(C_{i}^{\text {primary }}-C_{i}^{\text {duplicate }}\right)^{2}}$

$\mathrm{ARPD}=\frac{2}{n} \sum_{i=1}^{n} \frac{\left|C_{i}^{\text {primary }}-C_{i}^{\text {duplicate }}\right|}{\left(C_{i}^{\text {primary }}+C_{i}^{\text {duplicate }}\right)} \cdot 100 \%$

This approach, outlined in Dutton et al. (2009), provides an additional assessment of measurement uncertainty, and can be compared to the uncertainties calculated using propagation of error to understand if the propagation has captured most real sources of error. To calculate the ARPD, negative data were removed. In the future, zero replacement, or detection limit replacement for data with negative values, will be considered. The ARPD was then multiplied by the average pooled concentration measurements to get units of concentration that could be directly compared with the uncertainty estimates derived through propagation. This approach of using paired M-Pods does not necessarily incorporate error due to convection either, since the pair will generally have very similar airflow effects in both units. This is a limitation that should be studied further in this system.

\subsection{Validation and user study}

From 3 to 12 December 2012, and later from 17 to 22 January 2013, nine M-Pods were co-located with reference instruments at a Colorado Department of Public Health and Environment (CDPHE) air monitoring station in downtown
Denver. Total system performance was assessed by comparing laboratory-generated calibrations with calibrations based on "real-world" ambient data, referred to as collocation calibrations. This procedure may technically be sensor normalization, but we will refer to it as calibration here, as that is the practical purpose, and the mathematical procedure does not differ. Although less sophisticated, collocation calibration provides a practical and useful method of assessing sensor performance. The 2 nd collocation was performed with a fresh set of sensors and yielded slightly better results (Supplement). Reference instruments for calibration and validation were provided by CDPHE and the National Center for Atmospheric Research (NCAR). CO was measured using a Thermo Electron $48 \mathrm{c}$ monitor, $\mathrm{CO}_{2}$ and $\mathrm{H}_{2} \mathrm{O}$ were measured with a LI-COR LI-6262, $\mathrm{NO}_{2}$ was measured using a Teledyne 200E, and $\mathrm{O}_{3}$ was measured with a Teledyne 400E. The $\mathrm{CO}_{2}$ instrument was calibrated before the deployment (LICOR, 1996), while the others were span- and zero-checked daily as per CDPHE protocol. The M-Pods were positioned 8 feet from the sampling inlets. They operated continuously in a ventilated shelter on the roof of the facility.

In the user-study portion of the validation, nine M-Pods were carried for over 2 weeks, with three users each carrying two M-Pods. The objective of the user study was to understand M-Pod inter-variability and how they drift over time during personal usage. Therefore, the actual personal exposure results are deemed less important, and are found in the Supplement. The M-Pods were calibrated before and after the deployment using collocation calibrations, following the same procedures as described for the December and January collocations. They were collocated at the CDPHE monitoring site in downtown Denver for $\sim 1$ week before and after the user study. They were worn on the user's upper arm or attached to backpacks or bags, and were placed as close as possible to the breathing area when users were sitting or sleeping. Users also kept daily logs with location and activity information.

Measurement values are minute medians of the $1 / 10 \mathrm{~Hz}$ raw data. The raw data were filtered beforehand for electronic noise. Sensor-specific thresholds of two standard deviations on the differences between sequential values were used to identify and remove noise spikes. An upper bound threshold on sequential differences provided another layer of filtering for the noisiest data. To ensure that sensors were warmed up, 10 minutes of data were removed after poweron. Additional noise filtering was applied for the collocation tests due to a bad USB power supply. These data were filtered for noise by applying the Grubbs test for outliers to the differences between all the M-Pods and a "reference" M-Pod that displayed less electronic noise. Final data completeness for the first and second collocation deployments ranged from 74.5 to 90.1 , and 56.5 to $99.1 \%$, respectively. Data filtered from each deployment were then $0.4-10.9$ and $0.4-4.8 \%$. 
Table 1. Collocation calibration summary statistics for December collocation using the linear model from Eq. (3).

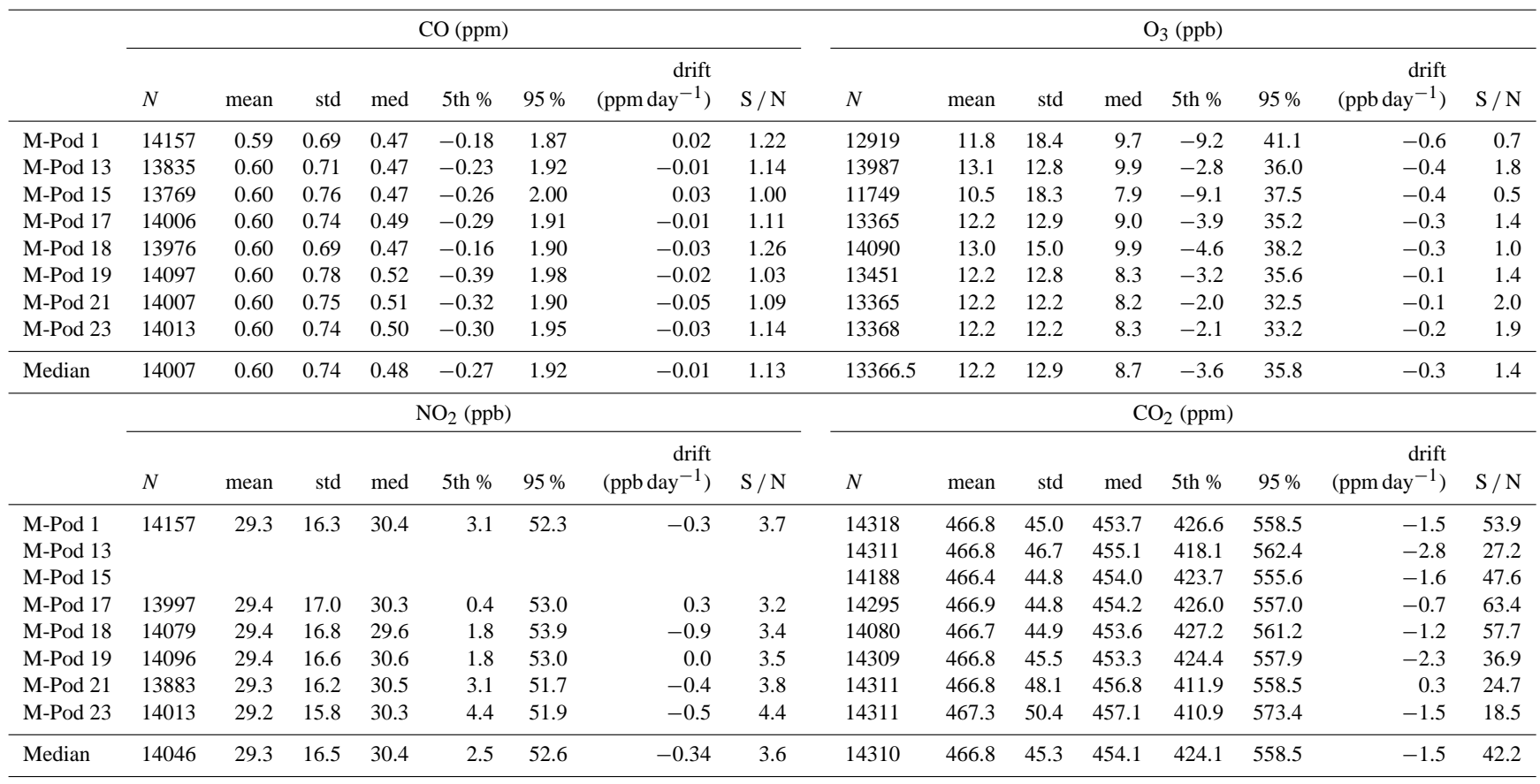

Table 2. Standard errors for the various calibration models tested with the December collocation data set. Equation (1), the exponential model, was not able to fit $\mathrm{O}_{3}$ satisfactorily for some unknown reason.

\begin{tabular}{|c|c|c|c|c|c|c|c|c|c|c|c|c|c|c|}
\hline \multirow[b]{2}{*}{ Model } & \multicolumn{5}{|c|}{$\mathrm{CO}_{2}(\mathrm{ppm})$} & \multicolumn{3}{|c|}{$\mathrm{CO}(\mathrm{ppm})$} & \multicolumn{3}{|c|}{$\mathrm{O}_{3}(\mathrm{ppb})$} & \multicolumn{3}{|c|}{$\mathrm{NO}_{2}(\mathrm{ppb})$} \\
\hline & Eq. $(6)^{b}$ & Eq. (6) w/time ${ }^{b}$ & Linear $^{b}$ & Linear $^{\mathrm{a}}$ & Eq. $(1)^{b}$ & Eq. $(2)^{b}$ & Eq. $(3)^{b}$ & Eq. $(1)^{\mathrm{a}}$ & Eq. $(1)^{b}$ & Eq. $(2)^{b}$ & Eq. $(3)^{b}$ & Eq. $(1)^{b}$ & Eq. $(2)^{b}$ & Eq. (3) \\
\hline M-Pod 1 & 8.4 & 7.3 & 11.0 & 138.7 & 0.4 & 0.38 & 0.38 & 3.69 & $\mathrm{NA}^{\mathrm{c}}$ & 15.4 & 14.9 & 7.2 & 8.2 & 8.2 \\
\hline M-Pod 13 & 16.8 & 14.4 & 18.2 & 29.1 & 0.4 & 0.42 & 0.41 & 3.54 & $\mathrm{NA}^{\mathrm{c}}$ & 5.6 & 5.4 & & & \\
\hline M-Pod 15 & 9.5 & 8.4 & 10.1 & 43.3 & 0.4 & 0.46 & 0.46 & 2.85 & $\mathrm{NA}^{\mathrm{c}}$ & 15.3 & 14.9 & & & \\
\hline M-Pod 17 & 7.2 & 6.9 & 15.5 & 15.2 & 0.4 & 0.44 & 0.44 & 3.22 & $\mathrm{NA}^{\mathrm{c}}$ & 6.4 & 6.2 & 7.5 & 9.5 & 9.5 \\
\hline M-Pod 18 & 7.9 & 7.1 & 10.5 & 30.0 & 0.3 & 0.38 & 0.37 & 3.58 & $\mathrm{NA}^{\mathrm{c}}$ & 9.8 & 9.6 & 7.9 & 9.0 & 8.8 \\
\hline M-Pod 19 & 12.3 & 10.4 & 31.4 & 125.3 & 1.8 & 0.52 & 0.51 & 4.49 & $\mathrm{NA}^{\mathrm{c}}$ & 5.8 & 5.8 & 7.0 & 8.6 & 8.6 \\
\hline M-Pod 21 & 18.5 & 18.6 & 22.4 & 105.0 & 0.7 & 0.49 & 0.47 & 3.42 & $\mathrm{NA}^{\mathrm{c}}$ & 4.2 & 4.1 & 6.9 & 8.0 & 7.9 \\
\hline M-Pod 23 & 24.8 & 23.9 & 48.8 & 93.5 & 0.4 & 0.45 & 0.44 & 5.33 & $\mathrm{NA}^{\mathrm{c}}$ & 4.4 & 4.4 & 6.0 & 6.9 & 6.8 \\
\hline Median & 10.9 & 9.4 & 16.8 & 68.4 & 0.4 & 0.45 & 0.44 & 3.56 & 23064.1 & 6.1 & 6.0 & 7.1 & 8.4 & 8.4 \\
\hline
\end{tabular}

\section{Results}

\subsection{Lab vs. collocation calibration results}

A summary of the results from the 3 to 12 December collocation and lab-calibrated data are presented in Tables 1 and 2. Table 1 shows summary statistics for the first collocation calibration, while Table 2 shows the performance for the different calibration methods and models.

\subsubsection{MOx sensor results}

The MiCS-5525 CO sensor was found to have substantially higher error using lab-calibrations versus collocation calibrations. As shown in Table 1, the median standard error for collocation calibration was $0.45 \mathrm{ppm}$ (range $0.38-0.52 \mathrm{ppm}$ ), while the median lab calibration standard error was $3.56 \mathrm{ppm}$ (range 2.85-5.33 ppm). Adding a linear time correction, as in Eq. (3), was found to improve the fit in most MOx sensor data sets. In this case, it improved the fit of the collocation calibrations slightly, giving a median standard error of $0.44 \mathrm{ppm}$ (range $0.38-0.51 \mathrm{ppm}$ ). The median standard error for the exponential-based model from Eq. (1) was $0.39 \mathrm{ppm}$ (range $0.34-1.78 \mathrm{ppm}$ ), but it actually provided a worse fit in some cases. The linear form of the equation, Eq. (2), is a good approximation of the exponential form shown in Eq. (1), likely because of the small environmental variable space spanned by the observed data. We have included residual plots (Fig. 3) to demonstrate model performance. Note the absence of a trend in these residual plots. 

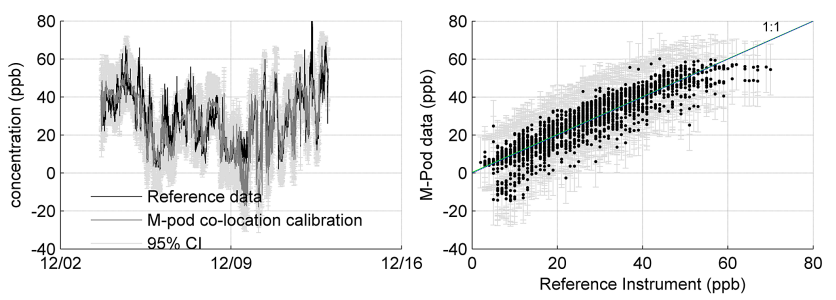

Figure 3. $\mathrm{NO}_{2}$ data from M-Pod 23 from the December collocation.

The relationship between collocation-calibrated sensor readings and reference data showed a slight negative bias at the higher end of observed concentration levels, but this appears to be driven by a small number of data points.

Inter-sensor variability is of interest if these sensors are to be widely deployed. Low variability could allow us to calibrate fewer sensors and apply those calibrations to other sensors in a large network. Inter-sensor variability for $\mathrm{CO}$ was generally low, with median correlation coefficients among the M-Pods 0.70 (range 0.62-0.78). The signal to noise $(\mathrm{S} / \mathrm{N})$ ratio, defined as the median observed value over the standard error, was 1.13 (range 1.00-1.26). This compares with the reference monitor $\mathrm{S} / \mathrm{N}$ of 10.0 , calculated using the median standard error from four days of zero and spancheck data from the monitor as the noise. The $\mathrm{S} / \mathrm{N}$ ratio provides straightforward comparison of instruments, and shows us how often the measurements are above the noise.

For the $\mathrm{O}_{3}$ and $\mathrm{NO}_{2}$ sensors, the model in Eq. (2) gave evenly distributed residuals and median standard errors of $6.1 \mathrm{ppb}$ (range 4.2-15.4 ppb), and $8.4 \mathrm{ppb}$ (range 6.9$9.5 \mathrm{ppb}$ ), respectively. As shown in Table 2, the linear model from Eq. (2) was found to fit the data nearly as well for $\mathrm{NO}_{2}$ as the non-linear model from Eq. (1), and is much less computationally intensive to use. An $\mathrm{NO}_{2}$ example time series using the linear model from Eq. (2) is shown in Fig. 3. The non-linear model was not able to fit the $\mathrm{O}_{3}$ data with any success, also shown in Table 2. The reason for this was not determined despite repeated testing. Lab calibrations were not performed for $\mathrm{O}_{3}$ and $\mathrm{NO}_{2}$. Median inter-sensor correlation for $\mathrm{O}_{3}$ was 0.83 (range 0.46-0.99), and 0.96 (range 0.940.99 ) for $\mathrm{NO}_{2}$. The median $\mathrm{NO}_{2} \mathrm{~S} / \mathrm{N}$ was 3.6 (range 3.34.4), compared with the median reference instrument $\mathrm{S} / \mathrm{N}$ of 23.4. For $\mathrm{O}_{3}$, the median $\mathrm{S} / \mathrm{N}$ ratio for the M-Pods was 1.4 (range $0.5-2.0$ ), while the reference instrument we collocated with had $\mathrm{S} / \mathrm{N}$ of 1.6. The reference instrument $\mathrm{S} / \mathrm{N}$ were calculated in the same way as for the $\mathrm{CO}$ monitor, using the median value of standard error from multiple days of zero and span data.

\subsubsection{NDIR $\mathrm{CO}_{2}$ sensor results}

$\mathrm{CO}_{2}$ values quantified with lab calibrations showed bias in some M-Pods (see Table 2), while others showed a high degree of accuracy. With collocation calibration, we also found

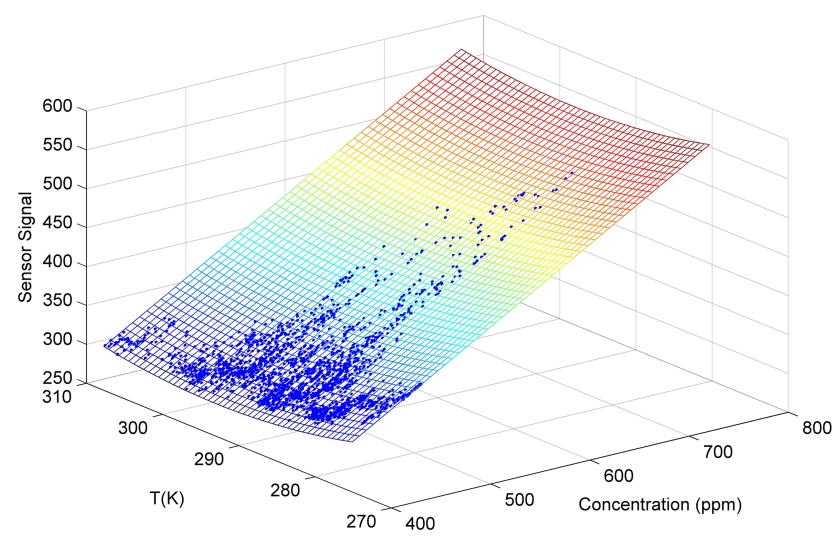

Figure 4. Calibration surface (using Eq. 4) for a $\mathrm{CO}_{2}$ collocation calibration performed from 3 to 12 December using M-Pod 1 .

a previously unseen temperature effect, described by

$v=p_{1}+p_{2} C+p_{3}\left(T-p_{4}\right)^{2}$,

where $v$ is the raw sensor signal. This model fit better than a linear model in concentration, and an example is shown in Fig. 4.

As shown in Table 2, when using linear models in concentration only, the median standard error for M-Pod $\mathrm{CO}_{2}$ measurements using the lab calibrations was $68.4 \mathrm{ppm}$ (range $15.2-138.7 \mathrm{ppm}$ ), and was $16.8 \mathrm{ppm}$ (range 10.1-48.8 ppm) using the collocation calibration. Median standard error was $10.9 \mathrm{ppm}$ (range 7.2-24.8 ppm) using the collocation calibration model from Eq. (6), and adding a linear time correction to this model further improved the fit, dropping the median standard error to $6.9 \mathrm{ppm}$. This drift term was statistically significant. The improvement in fit with the more complex model may be due to a temperature effect of the semiconductor infrared sensor, or an unidentified confounding variable. Adding humidity as a variable was not found to improve the fit significantly. Using the collocation calibration approach, the median correlation between $\mathrm{CO}_{2}$ sensors in different M-Pods was 0.88 (range 0.58-0.98). The median signal to noise ratio was 42.2 (range 18.5-63.4), as compared with a reported 300-500 from the reference instrument used (LI-COR, 1996).

\subsection{User-study results}

Based on initial lab and collocation calibration results, calibrations for the user-study were performed only with collocation calibrations. Collocation calibrations were carried out before and after the 3-week measurement period. Calibration fits were comparable to the prior collocation calibrations for $\mathrm{CO}$ (median standard error of $0.3 \mathrm{ppm}$ ), $\mathrm{NO}_{2}$ (median standard error of $8.8 \mathrm{ppb}$ ), and $\mathrm{O}_{3}$ (median standard error of $9.7 \mathrm{ppb}$ ). For $\mathrm{CO}_{2}$, the median standard error was high (36.9 ppm), likely because we were unable to colocate a reference monitor with the M-Pods at these times. 
Table 3. Average pooled uncertainty calculations for user study duplicate measurements.

\begin{tabular}{|c|c|c|c|c|c|c|c|c|c|}
\hline & \multicolumn{3}{|c|}{$\mathrm{CO}(\mathrm{ppm})$} & \multicolumn{3}{|c|}{$\mathrm{O}_{3}(\mathrm{ppb})$} & \multicolumn{3}{|c|}{$\mathrm{NO}_{2}(\mathrm{ppb})$} \\
\hline & $\begin{array}{l}\text { Propagated } \\
\text { uncertainty }\end{array}$ & $\mathrm{SD}_{\text {diff }}$ & ARPD & $\begin{array}{l}\text { Propagated } \\
\text { uncertainty }\end{array}$ & $\mathrm{SD}_{\text {diff }}$ & ARPD & $\begin{array}{l}\text { Propagated } \\
\text { uncertainty }\end{array}$ & $\mathrm{SD}_{\text {diff }}$ & ARPD \\
\hline M-Pod 6, 9 & 0.24 & 0.58 & $0.63(66.9 \%)$ & 7.9 & 15.5 & $20.6(59.8 \%)$ & 8.7 & 11.8 & $18.4(38.6 \%)$ \\
\hline M-Pod 15, 16 & 0.92 & 3.8 & $4.57(133 \%)$ & 14.6 & 17.1 & $18(80.8 \%)$ & 8.8 & 7.4 & $12.0(24.2 \%)$ \\
\hline M-Pod 23, 25 & 0.28 & 0.36 & $0.36(55.5 \%)$ & 11.2 & 25.7 & $12.8(53.3 \%)$ & 8.8 & 4.4 & $7.4(19.9 \%)$ \\
\hline
\end{tabular}

Instead, a calibration curve was generated using data from a nearby ambient monitor operated by NCAR, and a lab calibration. The monitor, located at the Boulder Atmospheric Observatory tower in Erie, Colorado, was used as reference for a nighttime period when ambient background concentration was assumed to be uniform over the region. Correlations among paired M-Pods during the user study ranged between 0.88 and 0.90 for $\mathrm{NO}_{2}, 0.48$ and 0.76 for $\mathrm{CO}, 0.33$ and 0.92 for $\mathrm{CO}_{2}$, and 0.04 and 0.35 for $\mathrm{O}_{3}$. The range of correlations for $\mathrm{CO}_{2}$ was due to power supply issues, which will be discussed later. We expect reliable $\mathrm{CO}_{2}$ sensor performance to be easily achievable in future work. Despite the low standard error from $\mathrm{O}_{3}$ sensor calibrations, we found low correlations among the paired M-Pods during the user study, which is also likely due to a power supply issue.

Measurement uncertainty calculated with the method of propagation and the duplicate M-Pod statistics, ARPD and

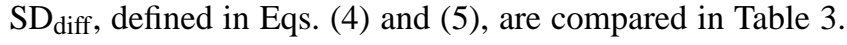
The results show moderate agreement among the methods for most pollutants. For $\mathrm{CO}$ and $\mathrm{O}_{3}$, the propagated uncertainty is lower than the $\mathrm{SD}_{\text {diff }}$ and $\mathrm{ARPD}$, roughly $50-75 \%$ of it, confirming that there are sources of error that are not accounted for in the uncertainty propagation. For $\mathrm{NO}_{2}$, the propagated measurement uncertainty seems to capture most of the uncertainty observed in the pairs. The RMSE values from the sensor calibrations were found to account for the majority of the propagated error. Figure 5 compares the $\mathrm{CO}$ measurements from M-Pods 23 and 25, along with their $95 \%$ confidence interval, the ARPD, and $\mathrm{SD}_{\text {diff }}$.

$\mathrm{S} / \mathrm{N}$ ratios during the user study were generally higher than during the collocations. This suggests that during personal exposure measurement, when concentration peaks are often higher than background measurements, the M-Pod is able to detect those peaks above the noise. Analysis based on the propagated uncertainty, $\mathrm{ARPD}$, and $\mathrm{SD}_{\text {diff }}$ suggests that propagated uncertainty is capturing most sources of error, but it does require more testing to further validate uncertainty estimation approaches. Personal exposure measurement results and discussion are shown in the Supplement.

Drift was seen to affect the measurement results, as described in detail in the Supplement. In the context of sensor work, drift is commonly considered to be deviations from anticipated or normal operation. These deviations are often directional rather than normally distributed (Ziyadtinov et al.,

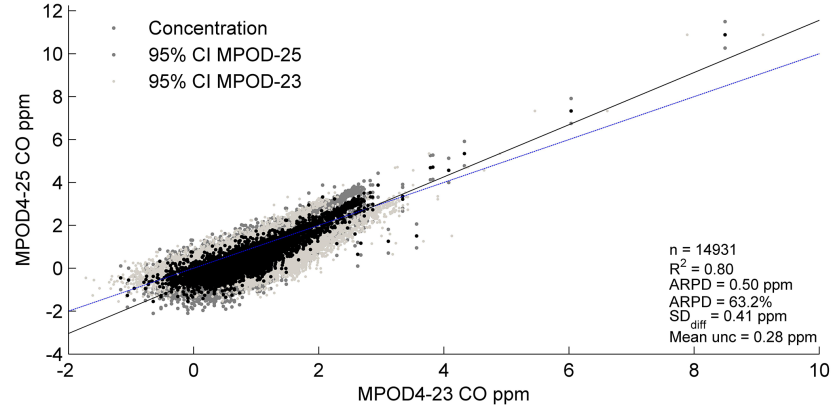

Figure 5. Personal CO measurement comparison between M-Pods 23 and 25, including $95 \%$ confidence intervals in light and dark gray, respectively.

2010), thus requiring more complex corrections. With this broad definition, drift includes confounding effects such as those due to temperature, humidity, pressure, and system error. This makes lab experimentation and fieldwork a challenging task with MOx sensors. Lab experiments must be designed to precisely control sensor temperature, humidity, gas concentration, flow conditions, etc. (Vergara et al., 2012). Even so, identifying mechanisms to cope with drift remain a significant challenge. Significant progress towards drift correction has been made in the domain of artificial machine learning (Di Natale et al., 2002; Vergara et al., 2012; Fonollosa et al., 2013; Martinelli et al., 2013).

For portable devices with limited computational power, or a widely distributed system where simplicity is preferable, it is advantageous to quantify the effect of drift through more direct means. We compensated for drift using multiple collocation calibrations with linear time corrections (Haugen et al., 2000), and observed improved calibration fits. Average daily drift during the user study is shown in Table S1 in the Supplement. For CO, all M-Pods experienced drift under $-0.05 \mathrm{ppm}_{\text {day }}{ }^{-1}$, apart from M-Pod 15, which showed behavior we cannot explain. $\mathrm{O}_{3}$ sensors experienced between -2.6 and $2.0 \mathrm{ppb}$ day $^{-1}$ drift. $\mathrm{CO}_{2}$ drift ranged from -4.2 to $3.1 \mathrm{ppm}^{-1 a y}{ }^{-1}$, excluding the bad results from M-Pod 9. $\mathrm{NO}_{2}$ generally showed a slight positive drift over time, with

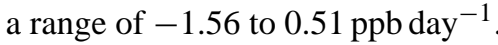




\section{Discussion}

The M-Pods performed well, given the relatively low ambient concentration environments encountered in the region. For $\mathrm{CO}, \mathrm{NO}_{2}$, and $\mathrm{CO}_{2}$, the reference instruments exhibited $\mathrm{S} / \mathrm{N}$ ratios 8-10 times higher than the M-Pod measurements. For $\mathrm{O}_{3}$, the reference monitor $\mathrm{S} / \mathrm{N}$ ratio was only slightly higher than the median M-Pod value.

\subsection{Lab calibration}

Lab calibrations had higher measurement error than collocation calibrations, likely because the field data covered a wider range of environmental variable space than the lab calibration. The poor field performance of lab calibrations may also be due to differences between the composition of zerograde air cylinders and ambient air. In this regard, filtered house air may be better suited to transfer calibrations out of the lab and to the field. Conducting field calibrations in the region of interest helps to account for confounding factors and meteorological variability.

$\mathrm{CO}_{2}$ lab-calibration results showed accurate results in some cases, while in other M-Pods, we found significant bias. Some $\mathrm{CO}_{2}$ sensors consistently showed poorer performance than others. Strangely, the poorer performing ones were usually in good agreement with each other. We have no explanation for this behavior, apart from possible sensor inconsistencies, or a potential power supply issue, addressed in more detail in the Supplement.

\subsection{Collocation calibration}

The time and resources required for lab calibration, and the difficulty reconciling the lab and ambient results, led us to rely more on collocation calibration. Collocation calibration performed well during two wintertime tests. However, during later collocation calibrations in warmer periods with rapidly changing weather, we found more interference from either reducing gases or humidity swings than we had previously seen. This effect, coupled with generally lower CO levels in the warmer months due to better atmospheric mixing and improved motor vehicle combustion (Neff, 1997), resulted in flatter and noisier calibration curves than previously seen. To minimize this effect, some portions $(10.1 \%)$ of the calibration data set were removed for April and May user study calibrations. $\mathrm{O}_{3}$ and $\mathrm{NO}_{2}$ had slightly worse calibration fits than during the winter calibrations, likely also due to larger swings in ambient humidity.

\section{Conclusions}

Collocation and collaborative calibration will be a valuable tool in the next generation of air quality monitoring. With help from monitoring agencies and citizen scientists, detailed ground-level pollutant maps will one day help track sources, reduce the population's exposure, and improve our knowledge of emissions as well as fate for each species. In this work, we have demonstrated a quantification system that can provide personal exposure measurements and uncertainties for $\mathrm{CO}_{2}, \mathrm{O}_{3}, \mathrm{NO}_{2}$, and $\mathrm{CO}$. This type of quantification approach provides access to air quality monitoring to a wider audience of scientists and citizens. A laboratory calibration system may cost thousands or tens of thousands of dollars, while a collocation calibration system only requires a decent enclosure for housing instruments. Whatever the application and precision requirements, investment to develop a calibration infrastructure, whether in a laboratory or near a monitoring station, is worthwhile in applications like health and exposure, source identification, and leak detection.

\section{The Supplement related to this article is available online at doi:10.5194/amt-7-3325-2014-supplement.}

Acknowledgements. This work was funded in part by CNSO910995, CNS-0910816, and CBET-1240584, from the National Science Foundation. Thank you to Bradley Rink and the Colorado Department of Public Health and Environment. Thank you to the Hannigan Lab members that made this work possible: Tiffany Duhl, Lamar Blackwell, Evan Coffey, Joanna Gordon, Nicholas Clements, and Kyle Karber.

Edited by: P. Di Carlo

\section{References}

Alphasense: O3-B4 Ozone Sensor Technical Specification, available at: http://www.alphasense.com/WEB1213/wp-content/ uploads/2013/11/O3B4.pdf (last access: 20 August 2014), 2013a.

Alphasense: NO2-B4 Ozone Sensor Technical Specification, available at: http://www.alphasense.com/WEB1213/wp-content/ uploads/2013/11/NO2B4.pdf, 2013b.

Barsan, N. and Weimar, U.: Conduction model of metal oxide gas sensors, J. Electroceram., 7, 143-167, 2001.

Bourgeois, W., Romain, A. C., Nicolas, J., and Stuetz, R. M.: The use of sensor arrays for environmental monitoring: interests and limitations, J. Environ. Monitor., 5, 852-860, 2003.

Colorado Department of Public Health and Environment Annual Data Report: available at: http://www.colorado.gov/airquality/ tech_doc_repository.aspx (last access: 29 September 2014), 2012.

Delpha, C., Siadat, M., and Lumbreras, M.: Humidity dependence of a TGS gas sensor array in an air-conditioned atmosphere, Sensor. Actuat.-B Chem., 59, 255-259, 1999.

De Vito, S., Piga, M., Martinotto, L., and Di Francia, G.: $\mathrm{CO}$, NO2 and NOx urban pollution monitoring with onfield calibrated electronic nose by automatic bayesian regularization, Sensors and Actuators B: Chemical, 143, 182-191, doi:10.1016/j.snb.2009.08.041, 2009. 
Di Natale, C., Martinelli, E., and D'Amico, A.: Counteraction of environmental disturbances of electronic nose data by independent component analysis, Sensor. Actuat.-B Chem., 82, 158-165, doi:10.1016/S0925-4005(01)01001-2, 2002.

Dutton, S. J., Schauer, J. J., Vedal, S., and Hannigan, M. P.: PM2.5 Characterization for Time Series Studies: Pointwise Uncertainty Estimation and Bulk Speciation Methods Applied in Denver, Atmos. Environ., 43, 1136-1146, 2009.

EPA ISA Health Criteria: US EPA National Center for Environmental Assessment, R.T.P.N., Long, T., Integrated Science Assessment for Carbon Monoxide (Final Report), available at: http: //cfpub.epa.gov/ncea/isa/recordisplay.cfm?deid=218686 (last access: 10 May 2014), 2010.

EPA ISA Health Criteria: US EPA National Center for Environmental Assessment, R.T.P.N., Brown, J., Integrated Science Assessment of Ozone and Related Photochemical Oxidants (Final Report), available at: http://cfpub.epa.gov/ncea/isa/recordisplay. cfm?deid=247492 (last access: 10 May 2014), 2013a.

EPA ISA Health Criteria: US EPA National Center for Environmental Assessment, R.T.P.N., Luben, T., Integrated Science Assessment for Oxides of Nitrogen - Health Criteria (Final Report), available at: http://cfpub.epa.gov/ncea/cfm/recordisplay. cfm?deid=194645 (last access: 10 May 2014), 2013 b.

Fine, G. F., Cavanagh, L. M., Afonja, A., and Binions, R.: Metal Oxide Semi-Conductor Gas Sensors in Environmental Monitoring, Sensors, 10, 5469-5502, 2010.

Fonollosa, J., Vergara, A., and Huerta, R.: Algorithmic mitigation of sensor failure: Is sensor replacement really necessary?, Sensor. Actuat.-B Chem., 183, 211-221, doi:10.1016/j.snb.2013.03.034, 2013

Gardner, J. W. and Bartlett, P. N.: A brief history of electronic noses, Sensors and Actuators B: Chemical, 18, 210-211, doi:10.1016/0925-4005(94)87085, 1994.

Hasenfratz, D., Saukh, O., Sturzenegger, S., and Thiele, L.: Participatory air pollution monitoring using smartphones, in: Proc. 1st Int'1 Workshop on Mobile Sensing: From Smartphones and Wearables to Big Data, 2012.

Haugen, J. E., Tomic, O., and Kvaal, K.: A calibration method for handling the temporal drift of solid state gas-sensors, Anal. Chim. Acta, 407, 23-39, 2000.

HEI: Traffic-related air pollution: a critical review of the literature on emissions, exposure, and health effects, Health Effects Institute, Boston, 2010.

Honicky, R. J., Mainwaring, A., Myers, C., Paulos, E., Subramanian, S., Woodruff, A., and Aoki, P.: Common Sense: Mobile Environmental Sensing Platforms to Support Community Action and Citizen Science, Human-Computer Interaction Institute, 2008

Jiang, Y., Li, K., Tian, L., Piedrahita, R., Yun, X., Mansata, O., Lv, Q., Dick, R. P., Hannigan, M., and Shang, L.: MAQS: a mobile sensing system for indoor air quality, in: Proceedings of the 13th International Conference on Ubiquitous Computing, UbiComp 2011, ACM, New York, NY, USA, 493-494, 2011.

Jiang, Y., Pan, X., Li, K., Lv, Q., Dick, R., Hannigan, M., and Shang, L.: ARIEL: automatic wi-fi based room fingerprinting for indoor localization, in: Proceedings of the 2012 ACM Conference on Ubiquitous Computing (UbiComp '12). ACM, New York, NY, USA, 441-450, doi:10.1145/2370216.2370282, 2012.
Kamionka, M., Breuil, P., and Pijolat, C.: Calibration of a multivariate gas sensing device for atmospheric pollution measurement, Sensors and Actuators B: Chemical, 118, 323-327, doi:10.1016/j.snb.2006.04.058, 2006.

Kate, S. K.: Engineering Mathematics - I. Technical Publications, ISBN:9788184317183, 2009.

Kaur, S., Nieuwenhuijsen, M. J., and Colvile, R. N.: Fine particulate matter and carbon monoxide exposure concentrations in urban street transport microenvironments, Atmos. Environ., 41, 47814810, 2007.

Korotcenkov, G.: Metal oxides for solid-state gas sensors: What determines our choice?, Mater. Sci. Eng. B-Adv., 139, 1-23, 2007.

LI-COR: LI 6262 CO2/H2O Analyzer Manual, available at: ftp: //ftp.licor.com/perm/env/LI-6262/Manual/LI-6262_Manual.pdf, 1996.

Martinelli, E., Magna, G., De Vito, S., Di Fuccio, R., Di Francia, G., Vergara, A., and Di Natale, C.: An adaptive classification model based on the artificial Immune system for chemical sensor drift mitigation, Sensor. Actuat.-B Chem., 177, 1017-1026, doi:10.1016/j.snb.2012.11.107, 2013.

Marco, S.: The need for external validation in machine olfaction: emphasis on health-related applications, Anal. Bioanal. Chem., 406, 3941-3956, doi:10.1007/s00216-014-7807-7, 2014.

MAQS Website: http://car.colorado.edu:443, last access: 2 May 2014.

Masson, N., Piedrahita, R., and Hannigan, M.: Approach for Quantification of Metal Oxide Type Semiconductor Gas Sensors Used for Ambient Air Quality Monitoring, Sensor. Actuat.-B Chem., accepted, 2014.

Mead, M. I., Popoola, O. A. M., Stewart, G. B., Landshoff, P., Calleja, M., Hayes, M., Baldovi, J. J., McLeod, M. W., Hodgson, T. F., Dicks, J., Lewis, A., Cohen, J., Baron, R., Saffell, J. R., and Jones, R. L.: The use of electrochemical sensors for monitoring urban air quality in low-cost, high-density networks, Atmos. Environ., 70, 186-203, doi:10.1016/j.atmosenv.2012.11.060, 2013.

Milton, R. and Steed, A.: Mapping Carbon Monoxide Using GPS Tracked Sensors, Environ. Monit. Assess., 124, 1-19, 2006.

Moseley, P. T.: REVIEW ARTICLE: Solid state gas sensors, Meas. Sci. Technol., 8, 223-237, 1997.

Neff, W. D.: The Denver Brown Cloud Studies from the Perspective of Model Assessment Needs and the Role of Meteorology, J. Air Waste Manage., 47, 269-285, 1997.

Röck, F., Barsan, N., and Weimar, U.: Electronic Nose: Current Status and Future Trends, Chem. Rev., 108, 705-725, 2008.

Romain, A. C. and Nicolas, J.: Long term stability of metal oxidebased gas sensors for e-nose environmental applications: An overview, Sensor. Actuat.-B Chem., 146, 502-506, 2010.

Romain, A.-C., Nicolas, J., and Andre, P.: In situ measurement of olfactive pollution with inorganic semiconductors?: Limitations due to humidity and temperature influence, available at: http://orbi.ulg.ac.be/handle/2268/16896 (last access: 29 September 2014), 1997.

Satish, U., Mendell, M. J., Shekhar, K., Hotchi, T., Sullivan, D., Streufert, S., and Fisk, W. J.: Is $\mathrm{CO}_{2}$ an Indoor Pollutant? Direct Effects of Low-to-Moderate $\mathrm{CO}_{2}$ Concentrations on $\mathrm{Hu}-$ man Decision-Making Performance, Environ. Health Persp., doi:10.1289/ehp.1104789, 2012.

Shum, L. V., Rajalakshmi, P., Afonja, A., McPhillips, G., Binions, R., Cheng, L., and Hailes, S.: On the Development of a Sen- 
sor Module for Real-Time Pollution Monitoring, in: Information Science and Applications (ICISA), 2011 International Conference, 1-9, 2011.

Sohn, J. H., Atzeni, M., Zeller, L., and Pioggia, G.: Characterisation of humidity dependence of a metal oxide semiconductor sensor array using partial least squares Sensor. Actuat.-B Chem., 131, 230-235, 2008.

Sundgren, H., Winquist, F., Lukkari, I., and Lundstrom, I.: Artificial Neural Networks and Gas Sensor Arrays: Quantification of Individual Components in a Gas Mixture, Meas. Sci. Technol., 2, 464, doi:10.1088/0957-0233/2/5/008, 1991.

Tsow, F., Forzani, E., Rai, A., Rui Wang, Tsui, R., Mastroianni, S., Knobbe, C., Gandolfi, A. J., and Tao, N. J.: A Wearable and Wireless Sensor System for Real-Time Monitoring of Toxic Environmental Volatile Organic Compounds, Sensors J., 9, 1734-1740, doi:10.1109/JSEN.2009.2030747, 2009.

Vergara, A., Vembu, S., Ayhan, T., Ryan, M. A., Homer, M. L., and Huerta, R.: Chemical gas sensor drift compensation using classifier ensembles, Sensor. Actuat.-B Chem., 166-167, 320329, doi:10.1016/j.snb.2012.01.074, 2012.

Vergara, A., Fonollosa, J., Mahiques, J., Trincavelli, M., Rulkov, N., and Huerta, R.: On the performance of gas sensor arrays in open sampling systems using Inhibitory Support Vector Machines, Sensor. Actuat.-B Chem., 185, 462-477, doi:10.1016/j.snb.2013.05.027, 2013.

Williams, D. E., Henshaw, G., Wells, D. B., Ding, G., Wagner, J., Wright, B., Yung, Y. F., Akagi, J., and Salmond, J.: Development of Low-Cost Ozone and Nitrogen Dioxide Measurement Instruments Suitable for Use in An Air Quality Monitoring Network, in: ECS Transactions, Presented at the 215th ECS Meeting, San Francisco, CA, 251-254, 2009.
Williams, D. E., Henshaw, G. S., Bart, M., Laing, G., Wagner, J., Naisbitt, S., and Salmond, J. A.: Validation of Low-Cost Ozone Measurement Instruments Suitable for Use in an AirQuality Monitoring Network, Meas. Sci. Technol., 24, 065803, doi:10.1088/0957-0233/24/6/065803, 2013.

Wolfrum, E. J., Meglen, R. M., Peterson, D., and Sluiter, J.: Metal Oxide Sensor Arrays for the Detection, Differentiation, and Quantification of Volatile Organic Compounds at Sub-Parts-perMillion Concentration Levels, Sensor. Actuat.-B Chem., 115, 322-329, doi:10.1016/j.snb.2005.09.026, 2006.

Yasuda, T., Yonemura, S., and Tani, A.: Comparison of the Characteristics of Small Commercial NDIR $\mathrm{CO}_{2}$ Sensor Models and Development of a Portable $\mathrm{CO}_{2}$ Measurement Device, Sensors, 12, 3641-3655, 2012.

Zakaria, R. A.: NDIR instrumentation design for Carbon Dioxide gas sensing, Doctoral dissertation, Cranfield University, available at: http://dspace.lib.cranfield.ac.uk/handle/1826/6784 (last access: 22 March 2014), 2010.

Zampolli, S., Elmi, I., Ahmed, F., Passini, M., Cardinali, G. C., Nicoletti, S., and Dori, L.: An Electronic Nose Based on Solid State Sensor Arrays for Low-Cost Indoor Air Quality Monitoring Applications, Sensor. Actuat.-B Chem., 101, 39-46, doi:10.1016/j.snb.2004.02.024, 2004.

Ziyatdinov, A., Marco, S., Chaudry, A., Persaud, K., Caminal, P., and Perera, A.: Drift compensation of gas sensor array data by common principal component analysis, Sensor. Actuat.-B Chem., 146, 460-465, doi:10.1016/j.snb.2009.11.034, 2010. 